\title{
Dynamic Behavior of Equivalent Cardiac Dipoles
}

Toshimitsu Musha, D.Sc., Yoshio OKamoto, B.Eng.

Yasua Ki Teramachi, D.Eng., and Kenichi Harumi, M.D*

T

HE body surface potentials are generated by excitation of heart muscle which is accompanied by development of electric dipole layers. The inverse problem eventually aims at finding how the excitation fronts are formed in the heart muscle.

Mathematically, however, an external potential distribution does not uniquely determine configuration of the excitation front but it probably does its peripheral shape, although no mathematical formulation has been obtained as yet which may give the peripheral shape of the excitation front in terms of the potential distribution on the body surface or on the epicardial surface.

We first tried to find a single equivalent cardiac dipole in terms of the potential distribution on the chest in such a way that squared

(a)

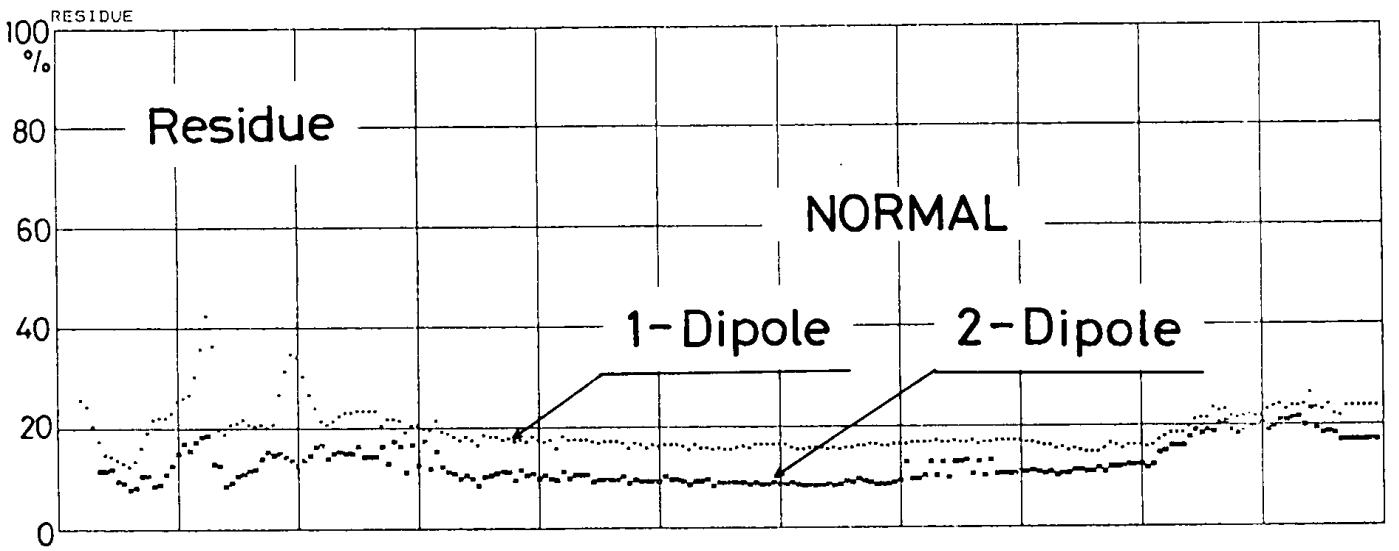

(b)

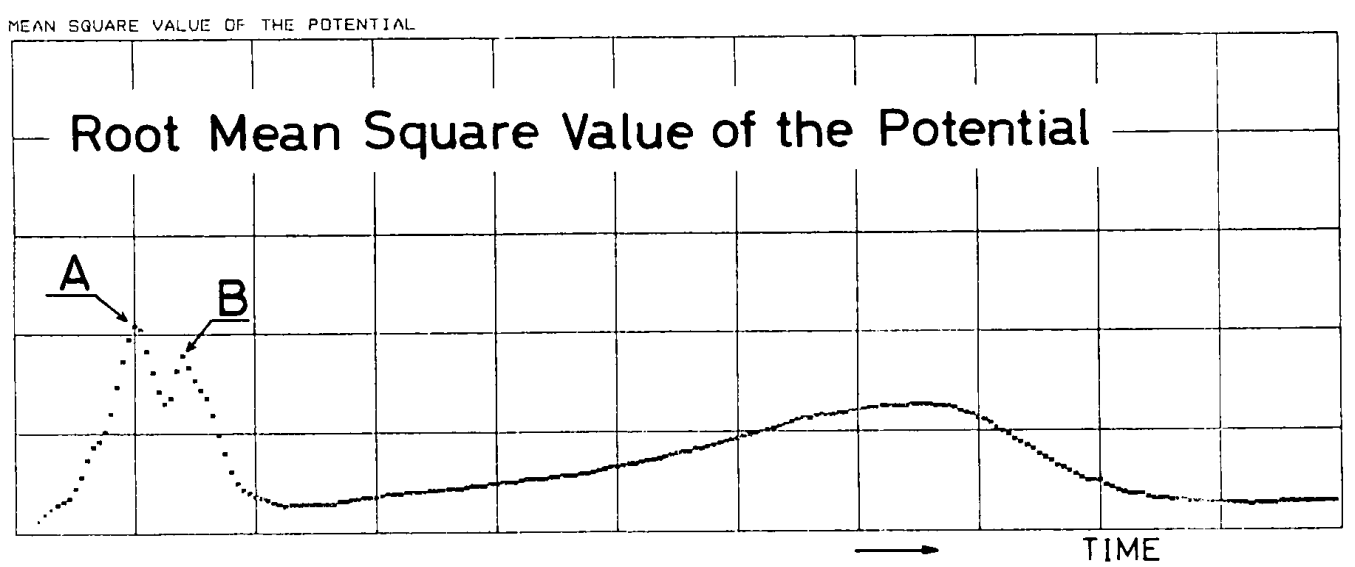

Fig.1.

Tokyo Institute of Technology, Nagatsuta, Midoriku, Yokohama 227, Japan

* Showa University, Fujigaoka Hospital 
(a)

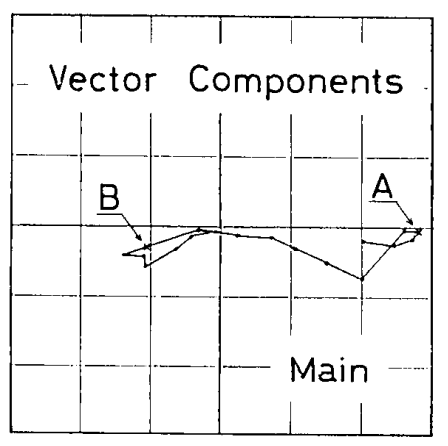

(b)

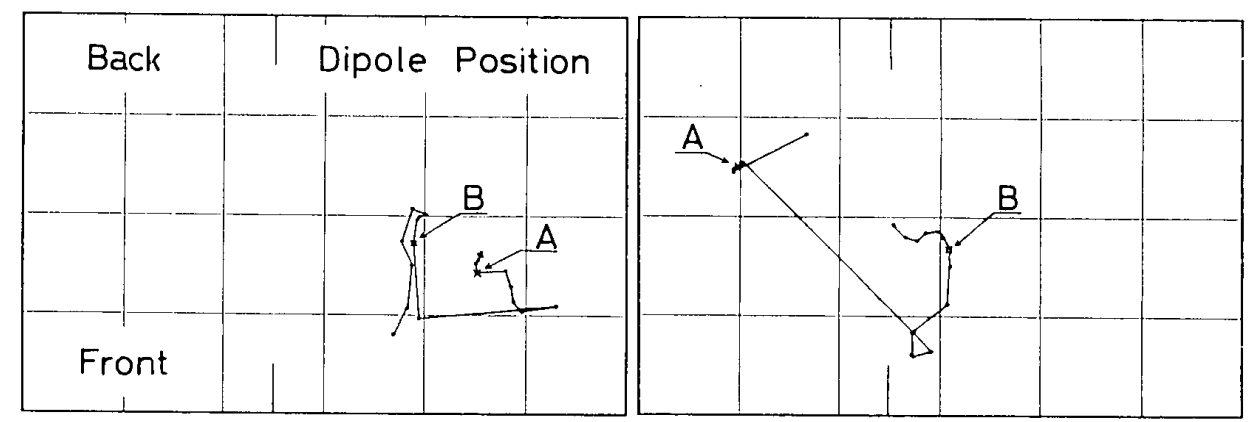

(c)

(d)

Fig.2.

deviation of the dipole potential on the body surface from the observed one, after being averaged over potentials measured on 64 electrode sites on the chest, was minimized.

Let the minimized root-mean-squared deviations be called "residue" when divided by a rootmean-square value of the potentials on the chest. A small residue would suggest that the approximation is good.

Figure 1(a) shows a residue in a single dipole approximation (indicated by '1-Dipole') of a normal subject. Figure 1(b) shows a root mean square of the body surface potentials averaged over the anterior chest, where two peaks, $\mathrm{A}$ and $B$, were observed. The residue reaches $40 \%$ in the QRS and stays about $20 \%$ elsewhere. This means that non-dipolar potential distribution is as large as $40 \%$ and $20 \%$, respectively.

In order to reduce the residue a step more, another equivalent dipole was introduced in addition to the original one, this we call the twodipole approximation. Their dipole moments and locations were determined such that the residue was minimized at every time point; the sampling time in the present observation was $2 \mathrm{~ms}$.

A curve in Fig. 1(a) which is indicated by "2. Dipole" is a residue in the two-dipole approxima- tion. The residue in this case is much smaller than the residue in the single-dipole approximation as was expected. Two peaks, which were found in the single dipole approximation in the QRS, are no longer found in the residue for the two-dipole approximation, and the residue as a whole remains at about $10 \%$.

Figures 2 (a) and (b) show dipole moment and location of a main dipole of the two which have been projected on the horizontal plane, the lower edges correspond to the front, and a unit length in (b) is equal to $4 \mathrm{~cm}$ which is an interval between the electrodes. Figures 2 (c) and (d) show dipole moment and location of a subdipole which have been projected on the horizontal plane.

In the first half of the QRS the main dipole was dominating and its role was overtaken by the subdipole in the last half of the QRS.

We are now very much interested in seeing whether one of the two dipoles stays at a location of infarction, if it exists, or not in the course of the QRS and T.

Question: How did you decide the main dipole? Answer: A dipole with a bigger dipole moment in the initial stage of the QRS was defined as a main dipole. 